\title{
Wireless Sensor Networks Topology Model Based on Energy Consumption and Life Cycle
}

\author{
https://doi.org/10.3991/ijoe.v14i05.8642 \\ Ruo Jia \\ Guizhou University of Commerce, Guiyang, China \\ $365530258 @$ qq. com
}

\begin{abstract}
Regarding the disadvantages of Wireless Sensor Networks such as random node failure, imbalanced network energy consumption, and shorter life cycle etc., the paper proposes an improved evolving model with an overall integration of fault-tolerance topology and network energy consumption, as a fusion mechanism optimizing the original node degree, generation ability and node distance in the model; it also validates the advantages of the improved model in terms of network life cycle, residual energy of node, node spacing and fault tolerance etc. by comparing the model with the optimized model of traditional sensor. With the establishment of WSNs energy consumption model, the paper evaluates the network life cycle and analyses the influence of node spacing and residual energy of node on WSNs, by adopting which as the fitness functions of the model, a scale-free fault tolerance topology evolving model with link deletion mechanism is constructed. The simulation comparison shows that the topology structure of the improved model has significant scale-free characteristics, a balanced network energy consumption and a long network life cycle; it can also effectively increase the fault tolerance and intrusion tolerance of network.
\end{abstract}

Keywords-wireless sensor networks, scale-free topology, fault tolerance, energy consumption, life cycle

\section{$1 \quad$ Introduction}

Wireless Sensor Networks (WSNs), as a new type of network system of data collection and transmission, has developed rapidly in recent years. Fig 1 shows the working mechanism of WSNs. The sensor node in the system can feed back the data in the collecting zone to the observer in the central control system, and send the command of the observer to the perceived object in the network covered zone. Now the wireless sensor is mainly applied in battlefield positioning, traffic system, deep-sea detection, and bioinformation collection [1-5]. 


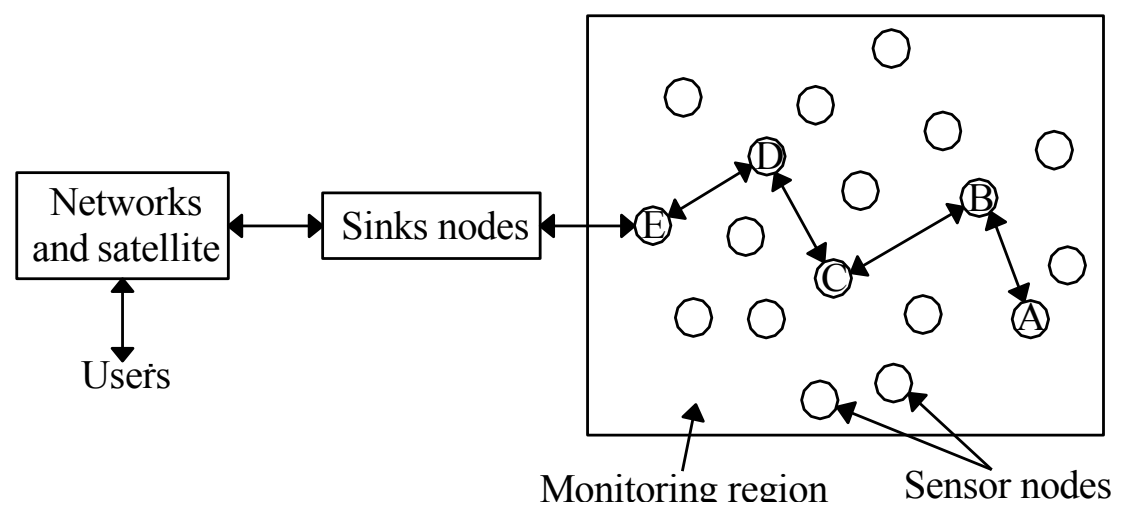

Fig. 1. The structure of Wireless Sensor Networks

The WSNs has certain limitations, incl. difficult replacement of the sensor node battery, node failure, higher energy consumption of the network system, and shorter network life cycle etc. resuting from the later start of the researches on WSNs. To make up for these limitations, this research proposes the model fault-tolerance technology and a scale-free network topology structure to improve the WSNs performance [6-14]. The frequently adopted method was to make improvements on the basis of traditional BA model, which enables the evolving topology of the model to satisfy the demands of different WSNs [15-20].

Regarding the disadvantages of WSNs such as random node failure, imbalanced network energy consumption, and shorter life cycle etc., the paper proposes an improved evolving model with an overall integration of fault-tolerance topology and network energy consumption, as a fusion mechanism optimizing the original node degree, generation ability and nodes distance in the model; it also validates the advantages of the improved model in terms of network life cycle, residual energy of node, node spacing and fault tolerance etc. by comparing the model with the optimized model of traditional sensor.

\section{WSNs energy consumption model and assessment method}

\subsection{WSNs life cycle assessment}

The WSNs life cycle is defined as the existential time of firstly-failed sensor node in the system. The energy consumption of the sensor system is closely related to the network life cycle, so the energy consumption model can be established to assess the network life cycle. With the energy of node perception and merging data far below that of data transmission and reception, the energy consumption model is mainly constructed on the basis of the consumed energy in data transmission and reception by the sensor node. The energy sending certain data to $d$ is given as:

$$
E(k, d)=E_{\text {elec }} k+k \varepsilon d^{\sigma}
$$


The energy consumption of receive node in receiving data is shown as:

$$
E(k)=E_{\text {elec }} k
$$

where $E_{\text {elec }}$ is the energy consumption for processing unit data; $\varepsilon d$ is the energy consumption of the amplifier in channel model; $\sigma$ is the communication path loss. In the two formulas above, the energy consumption at transmission terminal is more than that at receiving terminal, because the channel amplifier can lose some energy in the data transmission process and the amplifier power dissipation is related to data transmission distance. The formula is shown as:

$$
d_{0}=\sqrt{\frac{\varepsilon_{f s}}{\varepsilon_{a m p}}}
$$

where $\varepsilon_{f s}$ and $\varepsilon_{a m p}$ represent the power amplification coefficients of the free energy attenuation model and the multi-path attenuation model respectively. In the network system, the consumed energy of one node in single data transmission and reception is given as:

$$
E_{\mathrm{cos}}=2 E_{\text {elec }} k+k \varepsilon d^{\sigma}=\left\{\begin{array}{c}
2 E_{\text {elec }} k+k \varepsilon_{\text {fs }} d^{2}, \sigma=2 \\
2 E_{\text {elec }} k+k \varepsilon_{\text {amp }} d^{4}, \sigma=4
\end{array}\right.
$$

The total energy consumption of network system is as follows:

$$
E_{\text {costotal }}=\sum_{i=1}^{N} 2 E_{\text {elec }} k+k \varepsilon d_{i}^{\sigma}
$$

where $\mathrm{N}$ is the number of nodes in the system and $d_{i}$ is the distance between the neighbouring nodes. It can be seen in formula 5 that when $E_{\text {elec }}$ and $\mathrm{k}$ are known, the system energy consumption is only related to $d_{i}$. The energy consumption value of single node $i$ in the system can be shown as:

$$
E S(i)=\frac{E_{\mathrm{cos}}}{E_{r e}(i)}=\frac{2 E_{\text {elec }} k+k \varepsilon d_{i}^{\sigma}}{E_{r e}(i)}
$$

where $E_{r e}(\mathrm{i})$ is the residual energy of the node. Based on formula 3-6, the life cycle assessment formula of WSNs is calculated as:

$$
\text { LIFE }=\frac{1}{E S}=\frac{\overline{E_{r e}}}{E_{\text {costotal }}}=\frac{\sum_{i=1}^{N} E_{r e}(i) / N}{\sum_{i=1}^{N} 2 E_{\text {elec }} k+k \varepsilon d_{i}^{\sigma}}
$$


Formula 8 shows that $d_{i}$ and the mean value of the node residual energy are the main parameters of WSNs life cycle, which has an inverse relation with the former and a proportional relation with the latter. Life cycle is the key factor in the data collection and the processing of network nodes.

\subsection{Evolutionary analysis of the network scale-free topology}

The generation conditions for scale-free topology network are growth and preferential connection. The typical scale-free topology models include B model, EAEM model and FTEL model, which all only incorporate the fault-tolerance structure of scale-free model without considering the energy consumption of topology. Therefore, based on the optimized model of traditional WSNs topology structure, this paper proposes an improved evolving model (IBDB) with the overall integration with faulttolerance topology and network energy consumption, which optimizes the original node degree, generation ability and node distance in the model and ignores the link growth between old nodes. The evolutionary steps of the improved model are shown as:

Initialize the network, and set related calculating parameters;

Improve the growth mechanism; for every calculation, add the new network node and the connected edges $\mathrm{m}$ in total, and then connect the $\mathrm{m}$ edges to the existing nodes;

Adopt the preferential connection; the connection probability between the new added node and other nodes is calculated as:

$$
\pi\left(E, d, k_{i}\right)=\frac{F \cdot k_{i}}{\sum_{j} F_{j} \cdot k_{j}}
$$

where $\mathrm{F}$ as the fitness function is expressed as:

$$
F=\frac{E_{r e(i)}}{d_{i}}
$$

Then the residual energy of node in the model is given as:

$$
E_{r e(i)}=E_{0}-\mu k_{i}
$$

where $E_{0}$ is the energy of node at initial moment; $\mu$ is the related parameter for energy calculation. In formula (10), the relationship between node energy and node degree can be established.

Line deletion: along with the new added node and $m$ connecting lines, the existing $\mathrm{b}$ links in the network system shall be deleted. The anti-preferential probability $\pi^{*}(\mathrm{E}$, $\mathrm{d}, \mathrm{k}$ ) is expressed as: 


$$
\pi^{*}\left(E, d, k_{i}\right)=\frac{\left[1-\pi\left(E, d, k_{i}\right)\right]}{[N(t)-1]}=\frac{\left(1-\frac{E_{r e}(i) \cdot k_{i}}{d_{i} \cdot \sum_{j} E_{r e}(i) \cdot k_{i} / d_{j}}\right)}{[N(t)-1]}
$$

where $\mathrm{N}(\mathrm{t})$ is the network size at $\mathrm{t}$ calculated time. With less node energy and longer transmission distance, the connection line around this node can be deleted more probably; the formula (11) is used to ensure the deleted link balance the network consumption and extend life cycle.

\subsection{Dynamic characteristics of network topology evolution}

The dynamic topology evolutionary analysis is conducted for IBDB model. The degree distribution function means the probability that any network node contains $\mathrm{k}$ (number of edges) edges; the dynamical equation is expressed as:

$$
\frac{\partial k_{i}}{\partial t}=m \pi\left(E, d, k_{i}\right)-b \cdot\left[\pi^{*}\left(E, d, k_{i}\right)+\sum_{j \neq i} \pi^{*}\left(E, d, k_{j}\right) \pi^{*}\left(E, d, k_{i}\right)\right]
$$

After the prolonged topology evolution, the formula (12) can be converted into:

$$
\frac{\partial k_{i}}{\partial t} \approx \frac{m F k_{i}}{2(m-b) t \bar{F}}-\frac{2 b}{t}
$$

Due to the small initial mesh of sensor network, the average node degree is given as:

$$
\langle k\rangle=\frac{2(m-b) t}{m_{0}+t} \approx 2(m-b)
$$

Based on the three formulas above, $k_{i}(\mathrm{t})$ expression can be finally given as:

$$
k_{i}(t)=B\left(\frac{t}{t_{i}}\right)^{\beta}-B+m
$$

The dynamic parameter $\beta$ and correlation coefficient $B$ are expressed as:

$$
\begin{aligned}
& \beta=\beta(m, b)=\frac{m}{2(m-b)} \frac{F}{\bar{F}} \\
& B=B(m, b)=m-\frac{4 b(m-b)}{m} \cdot \frac{\bar{F}}{F}
\end{aligned}
$$


Then, the degree distribution form of the improved model is concluded as:

$$
p(k)=\frac{\partial p\left(k_{i}(t)<k\right)}{\partial k}=\frac{1}{\beta} \frac{t}{m_{0}+t} B^{\frac{1}{\beta}}(k-m+B)^{-\left(1+\frac{1}{\beta}\right)}=\frac{1}{\beta} \frac{t}{m_{0}+t} B^{\frac{1}{\beta}}(k-m+B)^{-\gamma}
$$

Above all, IBDB model can better realize the network scale-free topology, with great fault-tolerance performance.

\section{Simulation test and analysis}

By using Matlab programming, this chapter studies the degree distribution form of IBDM model, and then by comparing this model with B model, EAEM model and FTEL model makes a contrastive analysis in terms of the energy balance, WSNs life cycle, and invasion resistance of system mesh etc. The related parameters are set as follows: number of initial nodes 200 , mesh area $40,000 \mathrm{~m}^{2}$, max radius $70 \mathrm{~m}$, node output data package 200bit, and data fusion energy consumption $50 \mathrm{Nj} /$ bit. The test output result is the average of calculation results for 40 times; all nodes in the network have the same initial energy.

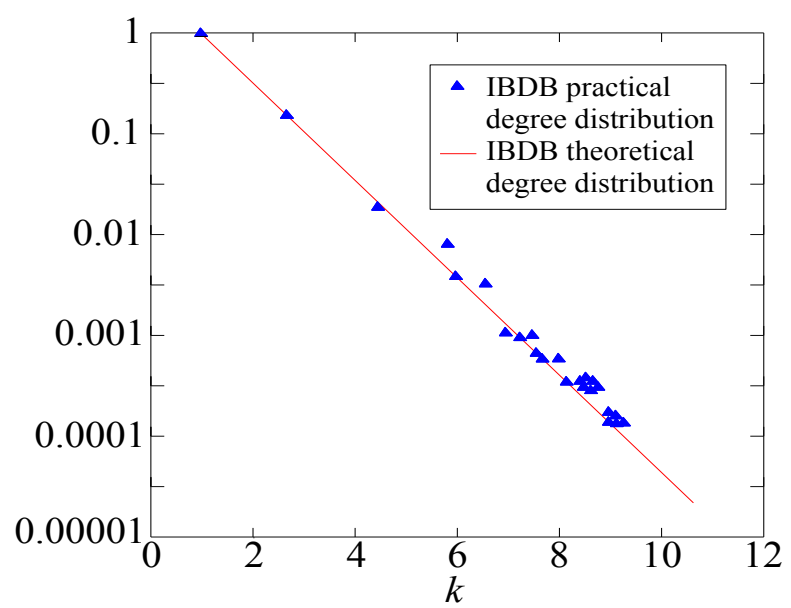

Fig. 2. Degree distribution of proposed model

Fig 2 depicts the curve of practical degree distribution and theoretical degree distribution in IBDB model, where $\mathrm{N}(\mathrm{t})=120$ and the degree distribution index $\gamma=3.5$. It's seen in Fig 2 that the degree distribution in IBDB model basically presents power-law distribution, and with a higher $\mathrm{k}$ value, the probability shall be less, indicating that the evolutionary process of IBDB model conforms to the scale-free topology conditions, and also indirectly to its strong fault-tolerance. 
Fig 3 depicts the node residual energy distribution of the selected EAEM model and IBDB model; the transverse line in Fig 3 means the average of the calculation results for 50 times.

Balanced energy consumption is one key reference index for WSNs life cycle. With the more balanced consumption energy for the sensor, the node shall not be more easily to fail, and then the life cycle of network system shall be longer. Fig 3(a) shows that the residual energy of traditional EAEM model system is $0.63 \mathrm{~J}$. By specifying $0.05 \mathrm{~J}$ as the energy interval, then it is found that the residual energy of almost half nodes is beyond the energy interval, with more singular nodes, e.g. over $0.75 \mathrm{~J}$ residual energy or about $0.5 \mathrm{~J}$ residual energy for some nodes, indicating that some nodes can easily fail due to excessive energy consumption, and some other nodes even has no energy consumption. EAME model doesn't fully integrate the relationship between energy and sensor network.

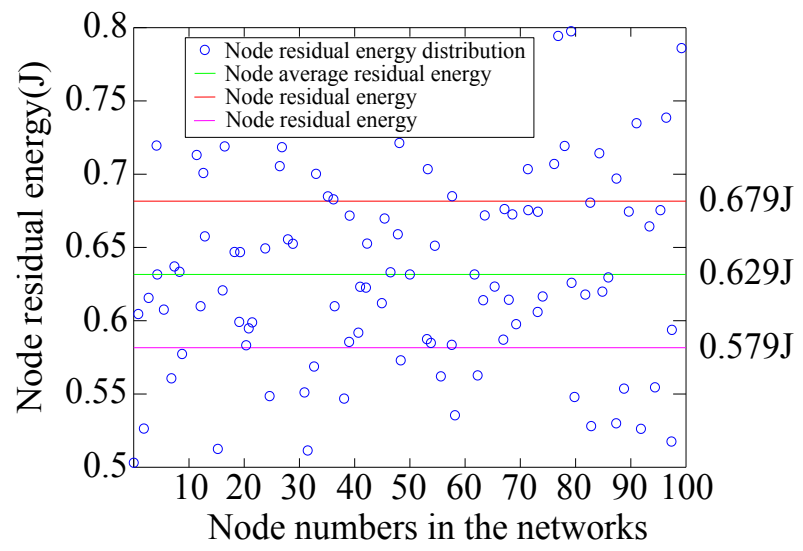

(a) Node residual energy of EAEM model

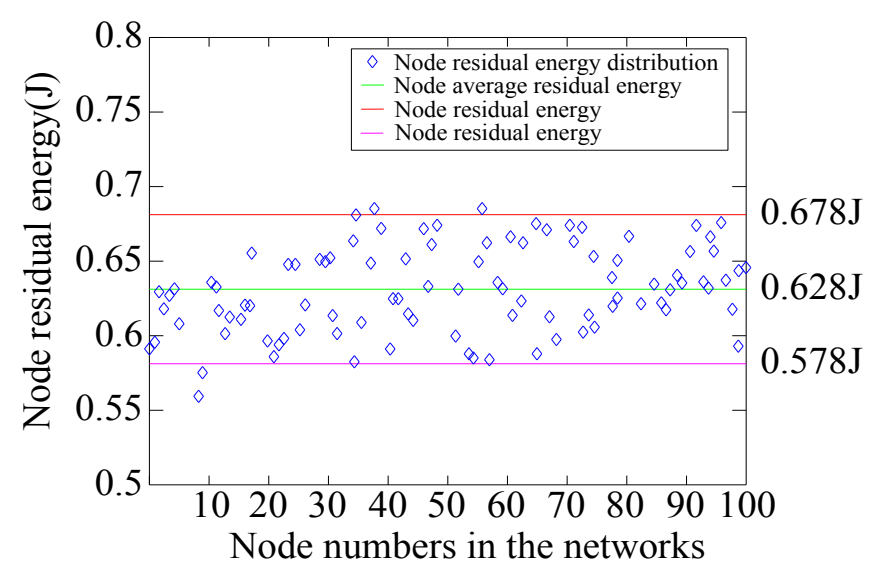

(b) Node residual energy of IBDB model

Fig. 3. Distribution chart of network node residual energy 
Fig 3(b) shows that the average of the node residual energy in IBDB model is $0.628 \mathrm{~J}$. By specifying the energy interval $\pm 0.05 \mathrm{~J}$, the residual energy of most nodes stays in the interval with balanced energy consumption, because in IBDM model, the preferential selection was set and also such factors as node degree, energy consumption, node spacing etc have been fully considered in the data transmission and receiving process. Furthermore, the link deletion mechanism of low-efficiency node in the model can ensure more energy-consuming links in the network system to be deleted in time, and keep the nodes with similar energy consumption. The analysis above has verified that the node energy consumption of scale-free topology structure is more balanced, and the model nodes within the same time are much fewer so as to better ensure the network system operation with more energy.

Network lifetime is the working time of WSNs in the whole data service period. Fig 4 depicts the accumulated lifetime of WSNs at different percentages of failure nodes for B model, EMEM model, FTEL model and IBDB model respectively. In every computation, data exchange and feedbacks were made between any node and its neighbouring nodes and the test result was the average of the calculated values for 30 times. Fig 4 shows when one node fails, the network lifetime in IBDB model reaches the maximum, then the EAEM model, FTEL, and finally the B model reaches the minimum because IBDB model has better balanced energy consumption and more energy can be used for the operation of network system. In a whole, IBDB model has the longest lifetime, far over that of the other three models. At the percentage of node failure $80 \%$, the accumulated lifetime of IBDB model is about 450 more than that of FTEL model, and about 600 more than that of EAEM, because during the preferential selection the IBDB model firstly selects the nodes with higher residual energy and shorter node spacing is connected with the new added nodes. Thus the scale-free network in topology evolution rule has better fault-tolerance.

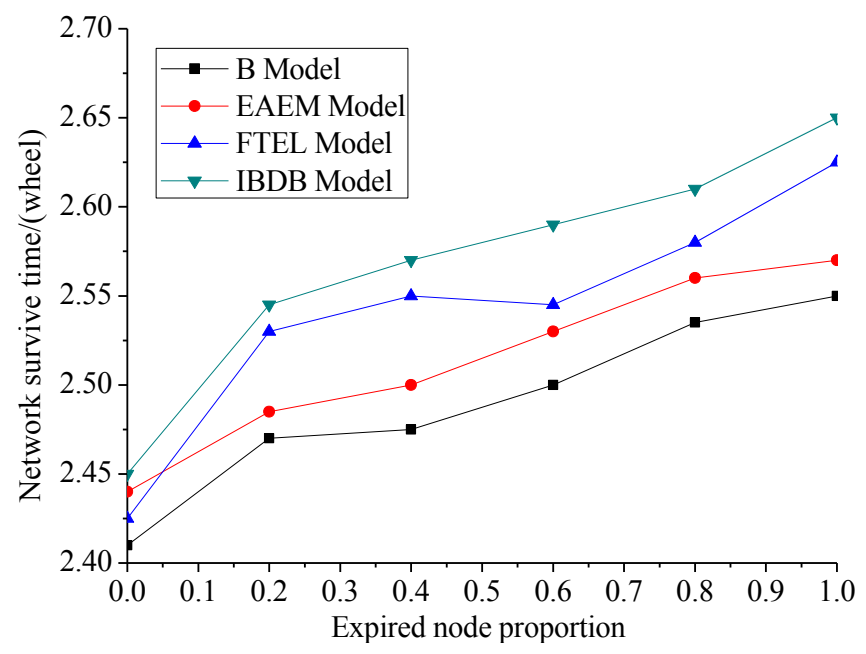

Fig. 4. Comparison results of network lifetime of four models 


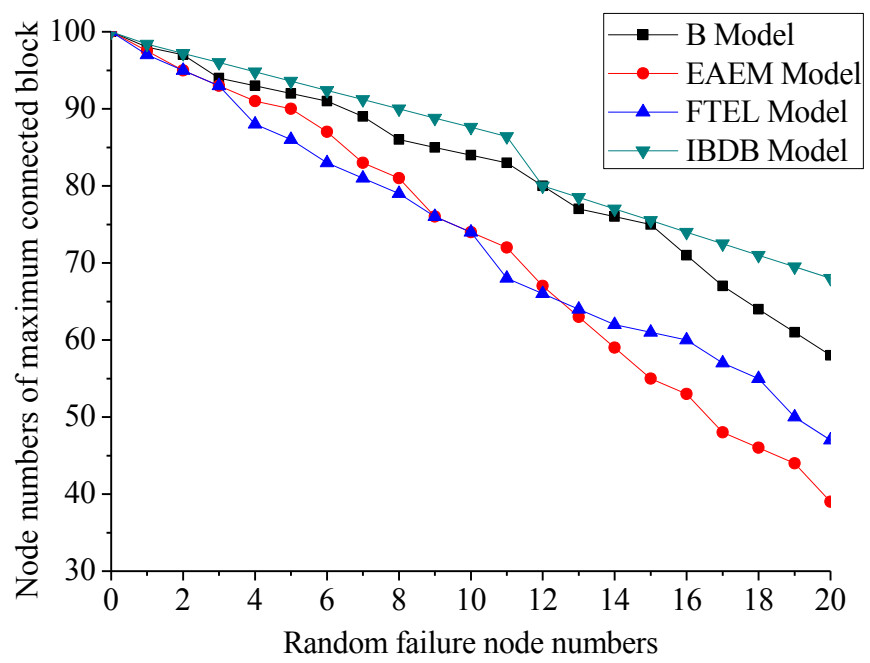

Fig. 5. Comparison results of fault tolerance capability of four models

It can be seen in Fig 5 that with the increasing number of failed nodes, the number of the nodes in the maximum connected region decreases continuously. The four models all present different degrees of fault-tolerance. The nodes of large nodedegree make up a small proportion of all nodes, while the nodes of small node degree occupy a large proportion. Therefore failure of small nodes has less influence on the network connectivity. Compared with the other three models, IBDB model has better performance in fault-tolerance: when the number of failed nodes reached 20, the maximum number of connected nodes was 70 , but 38, 47 and 60 respectively for EAEM model, FTEL model and B model repectively. The IBDB model proposed in this paper fully integrates the node residual energy and neighbouring nodes spacing with more reasonable node connection to reduce the quantity of failed nodes.

Fig 6 depicts the comparison of the intrusion tolerance performance among these four models. It can be seen in Fig 6 that the intrusion tolerance in IBDB is better than that in the other three models. In EAEM, FTEL, and B model, the nodes found no connected ones when the nodes were intruded and the failed nodes reached 7 , because in the scale-free topology structure the external intrusion into the core nodes has certain impact on the neighbouring nodes, reducing the number of connected nodes significantly. However, in IBDB model, the anti-preferential deletion mechanism is adopted to limit the network topology, avoiding adding new link randomly between the nodes. Above all, the IBDB has better performance in aspect of intrusion tolerance. 


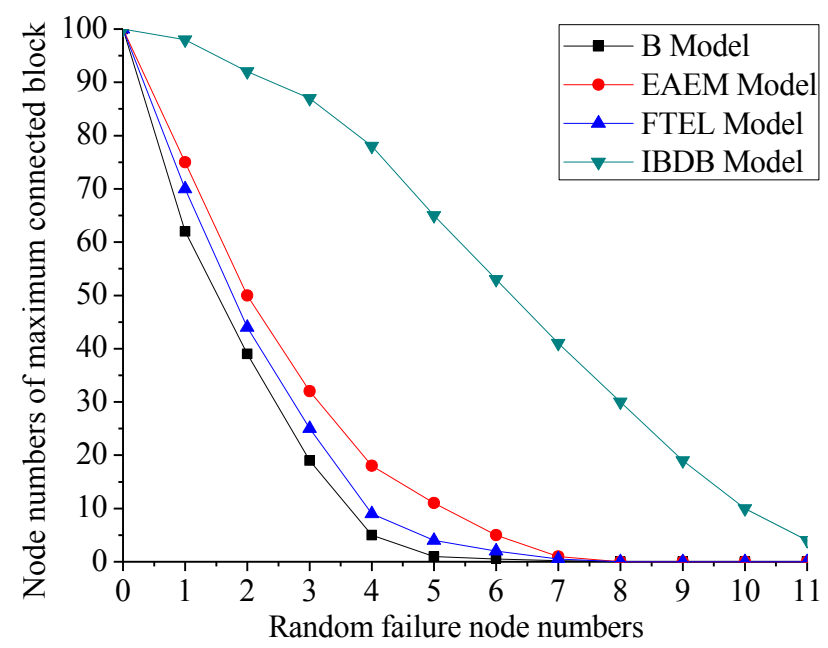

Fig. 6. Comparison results of tolerance invasion capability of four models

\section{Conclusion}

Regarding the disadvantages of WSNs such as random node failure, imbalanced network energy consumption, and shorter life cycle etc., the paper proposes an improved evolving model with an overall integration of fault-tolerance topology and network energy consumption, as a fusion mechanism optimizing the original node degree, generating ability and node distance in the model; it also validates the advantages of the improved model in terms of network life cycle, residual energy of node, node spacing and fault tolerance etc by comparing the model with the optimized model of traditional sensor. The conclusions are made as follows:

The paper, with establishment of WSNs energy consumption model, evaluates the network life cycle and analyses the influence of node spacing and residual energy of node on WSNs, by adopting which as the fitness functions of the model, a scale-free fault tolerance topology evolving model with link deletion mechanism is constructed.

The simulation comparison shows that the topology structure of the improved model has significant scale-free characteristics, a balanced network energy consumption and a long network life cycle; it can also effectively increase the fault tolerance and intrusion tolerance of network.

\section{$5 \quad$ References}

[1] Viani, F., Rocca, P., Oliveri, G., Trinchero, D., Massa, A. (2011). Localization, tracking, and imaging of targets in wireless sensor networks: an invited review, Radio Science, 46(5), 1161-1166. https://doi.org/10.1029/2010RS004561 
Paper-Wireless Sensor Networks Topology Model Based on Energy Consumption and Life Cycle

[2] Egbogah, E.E., Fapojuwo, A.O. (2011). A survey of system architecture requirements for health care-based wireless sensor networks, Sensors, 11(5), 4875-98. https://doi.org/10.3390/s110504875

[3] Li, H.M., Liu, H.H. (2017). The optimizated study on preparation process of nano tire pressure sensor used in auto, Academic Journal of Manufacturing Engineering, 15(1), 97104.

[4] Albaladejo, C., Sánchez, P., Iborra, A., Soto, F., López, J.A., Torres, R. (2010). Wireless sensor networks for oceanographic monitoring: a systematic review, Sensors, 10(7), 69486968. https://doi.org/10.3390/s100706948

[5] Banerjee, S., Ghosh, A., Mitra, S.K. (2017). A modified mathematical model for lifetime enhancement in wireless sensor network, Mathematical Modelling of Engineering Problems, 4(2), 84-90. https://doi.org/10.18280/mmep.040204

[6] Aziz, A.A., Sekercioglu, Y.A., Fitzpatrick, P., Ivanovich, M. (2013). A survey on distributed topology control techniques for extending the lifetime of battery powered wireless sensor networks, IEEE Communications Surveys \& Tutorials, 15(1), 121-144. https://doi.org/10.1109/SURV.2012.031612.00124

[7] Bredin, J.L., Demaine, E.D., Hajiaghayi, M.T., Rus, D. (2010). Deploying sensor networks with guaranteed fault tolerance, IEEE/ACM Transactions on Networking, 18(1), 216-228. https://doi.org/10.1109/TNET.2009.2024941

[8] He, Y., Zhang, W., Jiang, N., Li, L., Luo, X. (2015). The research of scale-free sensor network topology evolution based on cloud computing, Journal of Digital Information Management, 13(4), 266-275.

[9] Luo, X., Hu, Y., Zhu, Y. (2014). Topology evolution model for wireless multi-hop network based on socially inspired mechanism, Physica A Statistical Mechanics \& Its Applications, 416(C), 639-650. https://doi.org/10.1016/j.physa.2014.09.033

[10] Liu, H., Wan, P.J., Jia, X. (2005). Fault-tolerant relay node placement in wireless sensor networks, Lecture Notes in Computer Science, 3595(5), 230-239. https://doi.org/10.1007/ 1153371925

[11] Misra, S., Majd, N.E., Huang, H. (2014). Approximation algorithms for constrained relay node placement in energy harvesting wireless sensor networks, IEEE Transactions on Computers, 63(12), 2933-2947. https://doi.org/10.1109/TC.2013.171

[12] Jing, Y., Mai, X., Wei, Z., Baoguo, X. (2010). A multipath routing protocol based on clustering and ant colony optimization for wireless sensor networks, Sensors, 10(5), 4521-40. https://doi.org/10.3390/s100504521

[13] Newman, M.E.J. (2003). The structure and function of complex networks, Siam Review, 45(2), 167-256. https://doi.org/10.1137/S003614450342480

[14] Gao, Z.H. (2017). The driver's steering feel assessment using EEG and emg signals, NeuroQuantology, 16(1), 6-13.

[15] Dorogovtsev, S.N., Mendes, J.F. (2000). Scaling properties of scale-free evolving networks: continuous approach, Phys Rev E Stat Nonlin Soft Matter Phys, 63(2), 056125.

[16] Chen, Q., Shi, D. (2004). The modeling of scale-free networks, Physica A Statistical Mechanics \& Its Applications, 335(1), 240-248. (Chen and Shi,2004)

[17] Zhu, H., Luo, H., Peng, H., Li, L., Luo, Q. (2009). Complex networks-based energyefficient evolution model for wireless sensor networks, Chaos Solitons \& Fractals, 41(4), 1828-1835. https://doi.org/10.1016/j.chaos.2008.07.032

[18] Zheng, G., \& Liu, Q. (2012). Scale-free topology evolution for wireless sensor networks ?], Computers \& Electrical Engineering, 38(3), 643-651. _ https://doi.org/10.1016/j.comp eleceng.2011.11.024 
[19] Wang, Y. Q. (2012). Study on a model of topology evolution of wireless sensor networks among cluster heads and its immunization, Acta Physica Sinica, 61(9), 1321-1323.

[20] Zheng, G., Liu, S., Qi, X. (2011). Topology evolution for wireless sensor networks with restorability mechanism, Journal of Computational Information Systems, 7(11), 40984104.

\section{Author}

Ruo Jia male (August 1977); Master of software engineering; lecturer; research direction: computer application, Internet of things applications. 18 years of teaching experience in program design and development, Compiled and published several textbooks, several articles are published in the public publication.

Article submitted 30 March 2018. Final acceptance 05 May 2018. Final version published as submitted by the author. 